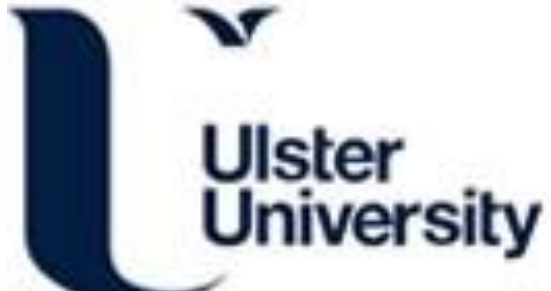

\section{Performance Analysis of Low-Complexity Uniform Power Loading with Reduced- Overhead OFDM Systems over Rayleigh Fading Channels}

Mohamed, E. B., \& Hossain, J. (2016). Performance Analysis of Low-Complexity Uniform Power Loading with Reduced-Overhead OFDM Systems over Rayleigh Fading Channels. 2015 IEEE 82nd Vehicular Technology Conference (VTC2015-Fall). https://doi.org/10.1109/VTCFall.2015.7391070

Link to publication record in Ulster University Research Portal

Published in:

2015 IEEE 82nd Vehicular Technology Conference (VTC2015-Fall)

Publication Status:

Published (in print/issue): 28/01/2016

DOI:

10.1109/VTCFall.2015.7391070

\section{Document Version}

Author Accepted version

\section{General rights}

Copyright for the publications made accessible via Ulster University's Research Portal is retained by the author(s) and / or other copyright owners and it is a condition of accessing these publications that users recognise and abide by the legal requirements associated with these rights.

\section{Take down policy}

The Research Portal is Ulster University's institutional repository that provides access to Ulster's research outputs. Every effort has been made to ensure that content in the Research Portal does not infringe any person's rights, or applicable UK laws. If you discover content in the Research Portal that you believe breaches copyright or violates any law, please contact pure-support@ulster.ac.uk. 


\title{
Short Paper: Performance Analysis of Low-Complexity Uniform Power Loading with Reduced-Overhead OFDM Systems over Rayleigh Fading Channels
}

\author{
Ebrahim Bedeer and Md. Jahangir Hossain \\ School of Engineering, University of British Columbia, Kelowna, BC, Canada \\ Email: \{ebrahim.bedeer-mohamed and jahangir.hossain\}@ubc.ca
}

\begin{abstract}
In this paper, we analyze the performance of two low-complexity uniform power loading with reduced-overhead orthogonal frequency division multiplexing (OFDM) schemes over Rayleigh fading channels. In the first feedback scheme, the receiver feeds back to the transmitter the channel gains and the indices of the best $M$ subchannels; while for the second feedback scheme, the receiver feeds back only the indices of the best $M$ subchannels. In both schemes, the available power budget is equally distributed over the best $M$ subchannels. We derive closed-form expressions for the capacity and the outage capacity of the first and second schemes, respectively. Numerical results show that there is an optimal number of the best subchannels, $M$, that maximizes the achievable capacity and it depends on the system parameters.
\end{abstract}

\section{INTRODUCTION}

The capacity of orthogonal frequency division multiplexing (OFDM) systems in frequency-selective channels can be maximized by adapting the transmit power per subchannel according to the well-known waterfilling (WF) solution [1]. However, optimal WF solutions need to be executed for every channel realization and require the feedback of all subchannels gains. In addition, designing the transmitter power amplifier becomes nontrivial due to the largely varying allocated powers among subchannels.

The capacity formula is a logarithmic function of the transmit power, and hence, the transmission rate is not very sensitive to the exact power allocation for subchannels with high gain. This motivates many researchers to develop suboptimal algorithms that distribute the power uniformly over subchannels with relatively high gains and null subchannels with relatively low gains, e.g., see [2]. However, all these algorithms requires processing for every channel realization, which increases the system complexity.

In this paper, we analyze the performance of two uniform power loading with reduced-overhead schemes over Rayleigh fading channels. In both schemes, no processing is required at the transmitter for every channel realization, which significantly reduces the system complexity. For the 1st scheme, the channel gains and the indices of the best $M$ subchannels are available at the transmitter; while for the 2nd scheme, only the indices of the best $M$ subchannels are known at the transmitter. We derive closed-form expressions for the capacity and outage capacity for the 1 st and 2 nd schemes respectively. Results show that there is an optimal number of the ordered subchannels, $M$, that can achieve up to $98.72 \%$ of the Shannon capacity limit. The analysis presented in the paper can assist to simplify the joint optimization problem of subchannels and power per subchannel of OFDMA systems.

\section{SYSTEM MODEL}

We consider an OFDM system that decomposes the signal bandwidth into a set of $N$ orthogonal narrowband subchannels each of equal bandwidth $W$. In the two schemes, the available power budget $S_{\max }$ is distributed equally over the best $M \leq N$ subchannels. The feedback channel for the first and second schemes requires $M b+N$ and $N$ bits, respectively, where $b$ is the number of bits required to feedback the channel gain of a certain subchannel. This is in contrast to $\mathrm{Nb}$ feedback bits required for optimal WF solutions.

Let us assume that the channel gains, $G_{1}, G_{2}, \ldots, G_{N}$, are sorted in a non-increasing order, i.e., $G_{(1)} \geq G_{(2)} \geq$ $\ldots \geq G_{(N)}$, where $G_{(n)}$ denotes the channel gain of the $n$th strongest subchannel gain. For independently and identically distributed fading channel, the joint probability density function (PDF) of the best $M$ subchannels can be written as

$$
\begin{aligned}
f_{G_{(1)}, G_{(2)}, \ldots, G_{(M)}}\left(g_{1}, g_{2}, \ldots, g_{m}\right)= & \frac{N !}{(N-M) !} F_{G}^{N-M}\left(g_{m}\right) \\
& \left(\prod_{n=1}^{M} f_{G}\left(g_{n}\right)\right), \quad(1)
\end{aligned}
$$

where $f_{G}\left(g_{n}\right)$ and $F_{G}\left(g_{n}\right)$ are the PDF and cumulative distribution function of the power gain of subchannel $n$, respectively.

\section{Performance AnAlysis of the Uniform Power LOADING SCHEMES}

Based on the joint PDF of the ordered subchannels in (1), the achievable capacity of the first scheme as a function of the ordered subchannel index $M$ can be written as in (2) on the top of the next page. Please note that $\frac{1}{\lambda}$ is the average channel gain, $\mathbf{E}_{1}(z)=\int_{z}^{\infty} e^{-t} t^{-1} d t$ is the exponential integral function, $\mathbf{B}(x, y)=\int_{0}^{1} t^{(x-1)}(1-t)^{(y-1)} d t$ is the Beta function, and $I, J, b_{i}, b_{j}, a_{I}$, and $a_{J}$ are defined as in [3].

In the 2nd scheme, outage is expected to occur as the channel gains of the best $M$ subchannels are not known at the transmitter. The outage capacity can be found as in (3) on the top of the next page. Please note that $\gamma(\alpha, x)=\int_{0}^{x} e^{-t} t^{\alpha-1} d t$ is the lower incomplete gamma function and $P_{\text {bound }}^{\text {out }}$ is an upper bound on the outage probability.

\section{Numerical and Simulation Results}

For numerical and simulation results, we consider an OFDM system with $N=8$ subchannels and $W=1 \mathrm{~Hz}$. The channel impulse response of length 5 taps is modeled as independent complex Gaussian random variable with zero mean and variance $\sigma^{2}$. Simulation results are averaged over $10^{4}$ channel realizations. 


$$
\begin{aligned}
& \eta_{\mathrm{C}}=\frac{W M^{2}}{\ln (2)}\left(\begin{array}{l}
N \\
M
\end{array}\right) \sum_{\ell=0}^{N-M}\left(\begin{array}{c}
N-M \\
\ell
\end{array}\right) \frac{(-1)^{\ell}}{(M+\ell)} \exp \left(\frac{\lambda M(M+\ell)}{S_{\max }}\right) \mathbf{E}_{1}\left(\frac{\lambda M(M+\ell)}{S_{\max }}\right) \\
& +4 \sqrt{2} \pi a_{I} a_{J} \frac{1}{\ln (2)} \frac{N !}{(N-M) !} \sum_{\tau=2}^{M} \frac{1}{(\tau-2) !} \exp \left(\frac{\lambda M(\tau-1)}{S_{\max }}\right) \\
& \cdot \sum_{j=1}^{J+1} \sum_{i=1}^{I+1} \mathbf{B}\left(M-(\tau-1)+4 b_{j} b_{i}(\tau-1), N-M+1\right) \prod_{\rho=1}^{M-\tau} \frac{1}{\left(\rho+4 b_{j} b_{i}(\tau-1)\right)} . \\
& \eta_{\mathrm{C}}^{\text {out }}=\left(1-P_{\text {bound }}^{\text {out }}\right) \eta_{\mathrm{C}}, \\
& P_{\text {bound }}^{\text {out }}=\left(\begin{array}{l}
N \\
M
\end{array}\right)\left[\frac{1}{(M-1) ! \gamma\left(M, \frac{2^{R_{\min }-1}}{S_{\max }} \lambda M^{2}\right)}+\sum_{q=1}^{N-M}(-1)^{M+q-1}\left(\begin{array}{c}
N-M \\
q
\end{array}\right) q^{1-M}\right. \\
& \left.\times\left(\frac{M}{M+q}\left(1-\exp \left(-\frac{2^{R_{\min }}-1}{S_{\max }}(M+q) \lambda M^{2}\right)\right)-\sum_{m=0}^{M-2} \frac{1}{m !}\left(-\frac{q}{M}\right)^{m} \gamma\left(m+1, \frac{2^{R_{\min }}-1}{S_{\max }} \lambda M^{2}\right)\right)\right]
\end{aligned}
$$

Fig. 1 depicts the achievable capacity of the 1 st scheme as a function of the ordered subchannel index for $S_{\max }=30 \mathrm{dBm}$ and average channel gains of $-5,0$, and $5 \mathrm{~dB}$. As can be seen, there is an optimal number of the most reliable subchannels that maximizes the capacity and this number depends on the system parameters, i.e., average channel gain and available power budget. More specifically, using $M=3,4$, and 6 subchannels out of 8 subchannels achieve $98.70 \%, 98.72 \%$, and $97.40 \%$ of the channel capacity obtained from the optimal WF solutions for average channel power gain of $-5,0$, and $5 \mathrm{~dB}$, respectively. Additionally, one can notice that for lower channel gains, less number of $M$ are required to approach the channel capacity. While, for higher channel gains, higher number of $M$ are required to approach the channel capacity. Fig. 1 interestingly reveals that fixed power allocation on an optimal $M$ subchannels is sufficient to approach the channel capacity obtained from optimal WF solutions, with reducedoverhead and less system complexity.

The performance of the 2nd scheme is plotted in Fig. 2 in terms of the outage probability $P_{\text {bound }}^{\text {out }}$. The optimal value of $M$ is obtained from (2) as in Fig. 1. As can be seen from Fig. 2, the outage probability significantly decreases for larger values of the power budget and larger channel power gain. Fig. 2 helps to control the quality of service given a set of the system parameters. For instance, an outage probability less than $10^{-4}$ can be achieved, if the receiver feeds back the location of the best 7 subchannels, without their actual channel gains, for $S_{\max }=20$ and $30 \mathrm{dBm}$.

\section{CONCLUSION}

In this paper, we analyzed the performance of two lowcomplexity uniform power loading with reduced-overhead OFDM schemes over Rayleigh fading channels. Both schemes requires no processing at the transmitter for every channel realization, which significantly reduces the system complexity. We derived closed-form expressions for the capacity and outage capacity for the 1 st and 2 nd schemes, respectively. Numerical results showed that the optimal number of the ordered subchannels $M$ depends on the system parameters, i.e., average channel gain and available power budget. For

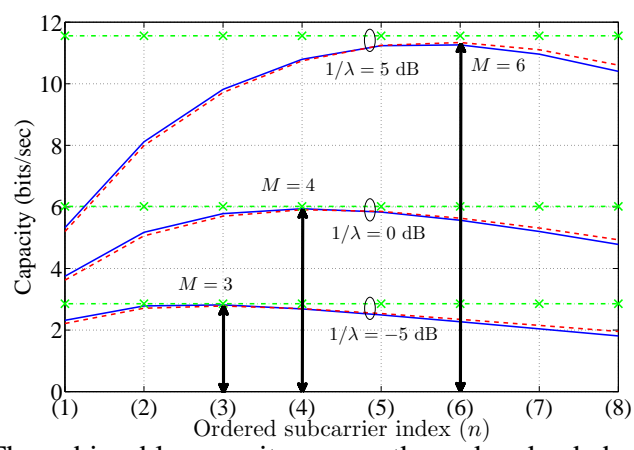

Fig. 1: The achievable capacity versus the ordered subchannel index for $S_{\max }=30 \mathrm{dBm}$ and average channel gains of $-5,0$, and 5 $\mathrm{dB}$. Solid blue lines represents numerical expression in (2), dash red lines represents simulation results, and dash-dot green line (with $\times$ marker) renresents the canacitv obtained from ontimal WF solutions.

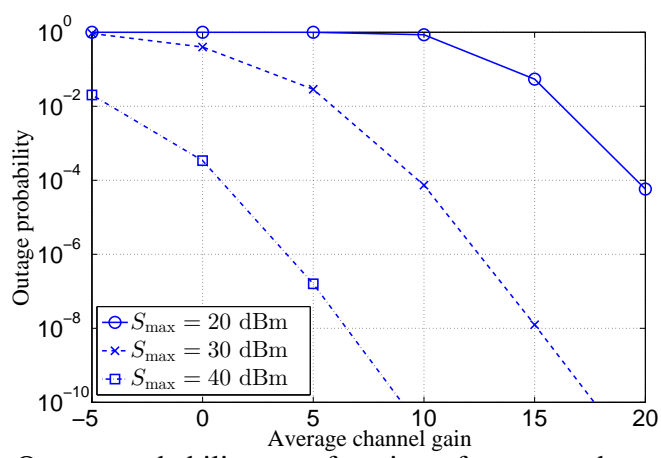

Fig. 2: Outage probability as a function of average channel gain for $R_{\text {min }}=1 \mathrm{bits} / \mathrm{sec} / \mathrm{Hz}$ and $S_{\max }=20,30$, and $40 \mathrm{dBm}$.

example, using as low as the 3 best subchannels can reach up to $98.72 \%$ of the channel capacity. The analysis presented in this paper can assist to simplify the design of OFDMA systems.

\section{REFERENCES}

[1] T. Cover and J. Thomas, Elements of Information Theory. Wiley, New York NY, 2004.

[2] W. Yu and J. M. Cioffi, "Constant-power waterfilling: performance bound and low-complexity implementation," IEEE Trans. Commun., vol. 54, no. 1, pp. 23-28, Jan. 2006.

[3] A. A. Alkheir and M. Ibnkahla, "An accurate approximation of the exponential integral function using a sum of exponentials," IEEE Commun. Lett., vol. 17, no. 7, pp. 1364-1367, Jul. 2013. 